\title{
Insights into the genome architecture and evolution of Shiga toxin encoding bacteriophages of Escherichia coli
}

\author{
Graça Pinto ${ }^{1,2}$, Marta Sampaio ${ }^{1}$ Oscar Dias ${ }^{1}$, Carina Almeida², Joana Azeredo ${ }^{1 *}$ and Hugo Oliveira ${ }^{\text {** }}$
}

\begin{abstract}
Background: A total of 179 Shiga toxin-producing Escherichia coli (STEC) complete genomes were analyzed in terms of serotypes, prophage coding regions, and stx gene variants and their distribution. We further examined the genetic diversity of Stx-converting phage genomes (Stx phages), focusing on the lysis-lysogeny decision and lytic cassettes.

Results: We show that most STEC isolates belong to non-0157 serotypes (73\%), regardless the sources and geographical regions. While the majority of STEC genomes contain a single stx gene (61\%), strains containing two $(35 \%)$, three $(3 \%)$ and four (1\%) stx genes were also found, being stx2 the most prevalent gene variant. Their location is exclusively found in intact prophage regions, indicating that they are phage-borne. We further demonstrate that Stx phages can be grouped into four clusters (A, B, C and D), three subclusters (A1, A2 and A3) and one singleton, based on their shared gene content. This cluster distribution is in good agreement with their predicted virion morphologies. Stx phage genomes are highly diverse with a vast number of 1,838 gene phamilies (phams) of related sequences (of which 677 are orphams i.e. unique genes) and, although having high mosaicism, they are generally organized into three major transcripts. While the mechanisms that guide lysis-lysogeny decision are complex, there is a strong selective pressure to maintain the stx genes location close to the lytic cassette composed of predicted SAR-endolysin and pin-holin lytic proteins. The evolution of STEC Stx phages seems to be strongly related to acquiring genetic material, probably from horizontal gene transfer events.

Conclusions: This work provides novel insights on the genetic structure of Stx phages, showing a high genetic diversity throughout the genomes, where the various lysis-lysogeny regulatory systems are in contrast with an uncommon, but conserved, lytic system always adjacent to stx genes.
\end{abstract}

Keywords: STEC, Shiga toxin-encoding bacteriophages, Genomes, Clusters

\section{Background}

Shiga toxin-producing Escherichia coli (STEC) are important foodborne pathogens, responsible for numerous infections worldwide. STEC infections can progress into serious conditions, such as hemorrhagic colitis or hemolytic-uremic syndrome (HUS), which might lead to

\footnotetext{
*Correspondence: jazeredo@deb.uminho.pt; hugooliveira@deb.uminho.pt ${ }^{1}$ CEB - Centre of Biological Engineering, University of Minho, 4710-057 Braga, Portugal

Full list of author information is available at the end of the article
}

the patient's death [1]. In 2018, there were 8,658 confirmed infections in the European Union, and 11 of the 5,254 known outcomes resulted in the patient's death, which represent a fatality rate of $0.2 \%$ [2].

STEC strains are characterized by their ability to produce Shiga toxins, considered the major virulence factor of this pathotype. There are two known Shiga toxin types, Stx1 and Stx2, being further divided into three (a, c, d) and nine (a to i) subtypes, respectively [3-6]. The disease outcome is dependent on the Shiga toxin

(c) The Author(s). 2021 Open Access This article is licensed under a Creative Commons Attribution 4.0 International License, which permits use, sharing, adaptation, distribution and reproduction in any medium or format, as long as you give appropriate credit to the original author(s) and the source, provide a link to the Creative Commons licence, and indicate if changes were made. The images or other third party material in this article are included in the article's Creative Commons licence, unless indicated otherwise in a credit line to the material. If material is not included in the article's Creative Commons licence and your intended use is not permitted by statutory regulation or exceeds the permitted use, you will need to obtain permission directly from the copyright holder. To view a copy of this licence, visit http://creativecommons.org/licenses/by/4.0/ The Creative Commons Public Domain Dedication waiver (http://creativecommons.org/publicdomain/zero/1.0/) applies to the data made available in this article, unless otherwise stated in a credit line to the data. 
subtype carried by STEC strains, being believed that Stx2a is the most associated with severe forms of the disease [7-11]. Several STEC serotypes have been associated with disease; however, not all are linked with severe infections. The most relevant serotypes in health risk are O157, O26, O45, O91, O103, O104, O111, O113, O145, and $\mathrm{O} 121[12,13]$.

E. coli acquires Shiga toxin genes through a lambdoid prophage insertion, known as Shiga or Stx phage. Stx phages are temperate, meaning that their genomes are inserted into the bacterial chromosome upon infection [14]. However, their ability to excise and infect other hosts, that could occur at the gastrointestinal tract [15], makes them important drivers of horizontal gene transfer (HGT) of stx genes among E. coli serotypes and other members of Enterobacteriaceae family [16]. This ability to quickly gain, lose or exchange genes through Stx phages has a high impact on the pathogenicity profile and evolution of STEC strains.

From the early moments, Stx phages have been compared to the Lambda phage, the prototype of Lambdoid phages [17]. Stx phages are known to share similar morphologies, e.g. short or long non-contract tails [16, 18]. Their genomes spanning from 30 to $70 \mathrm{~kb}$, share little homology, although having a similar genetic organization [17]. Only a limited number of genomic studies have been performed so far with Stx phages, usually not including a vast number of genomes, nor considering their hosts (STEC serotypes) and environmental sources [1, 19-22]. Moreover, previous studies demonstrated that the analysis of shared gene content provides a more powerful tool to uncover distant relationships between viral sequences [23-25]. However, this has only been attempted for a small number of STEC Stx phages [26]. Currently, with the advance of sequencing platforms and higher availability of complete STEC genomes, genomic studies can evolve to the new level. For a better understanding of the impact of Stx phages in the STEC ecology, we performed an in-depth genomic study of all available Stx phages, to evaluate their genome diversity and organization, gene composition, as well as their association with specific STEC serotypes.

\section{Results}

\section{Multiple serotypes carry Stx virulence factors}

The well-known heterogeneity of the $E$. coli species is seen in the complete genomes retrieved from the database, with a vast diversity of $\mathrm{O}$ and $\mathrm{H}$ antigens (additional file 1). From the $787 \mathrm{E}$. coli genome sequences, 179 were identified as STEC, containing one or more $s t x$ genes. Within the STEC group, several strains belong to the O157:H7 serotype $(n=48,27 \%)$. This is not surprising since this serotype has been regarded as the most problematic in the context of STEC-associated foodborne infections, which has probably triggered a special attention on genomic studies for these strains. The high prevalence of this serotype was followed by O26:H11 ( $n=13,7.3 \%)$, O111:H8 ( $n=13,7.3 \%)$, O104: H4 $(n=13,7.3 \%), \mathrm{O} 145: \mathrm{H} 28(n=7,3.9 \%), \mathrm{O} 121: \mathrm{H} 19$ $(n=6,3.4 \%), \mathrm{O} 113: \mathrm{H} 21 \quad(n=4,2.2 \%)$ and $\mathrm{O} 177: \mathrm{H} 25$ $(n=4,2.2 \%)$. The other 50 serotypes are less represented $(\leq 2.0 \%)$.

STEC genomes carrying stx 2 genes are more common $(n=161 / 179,90 \%)$ than those carrying $\operatorname{stx} 1$ genes $(n=$ $100 / 179,56 \%)$. Of all $s t x$ gene variants detected in the $E$. coli dataset, the most common is the $\operatorname{stx} 2 a(n=106 / 260$, $41 \%)$, followed by $\operatorname{stx} 1 a(n=93 / 260,35 \%)$, and $s t x 2 c$ $(n=39 / 260,15 \%)$, whereas the remaining variants are less prevalent $(\leq 2.7 \%)$ (Fig. 1a). Strains carrying only one stx gene are the most common $(n=109,61 \%)$, followed by strains carrying two stx genes $(n=63,35 \%)$. Moreover, $4 \%$ of the bacterial genomes carry at least three stx genes (Fig. 1b). Curiously, one strain carries four stx $2 a$ genes. The most common combinations of stx genes are stx $1 a / s t x 2 a(n=35,20 \%)$, stx $2 \mathrm{a} /$ st $x 2 \mathrm{c}(n=$ $12,6.7 \%)$ and $s t x 1 \mathrm{a} / \mathrm{stx} 2 \mathrm{c}(n=9,5.0 \%)$. Genes stx2e, $s t x 2 f$ or $s t x 2 g$ were not combined with other $s t x$ variants. Most STEC strains were detected in humans $(n=112$, $63 \%)$, followed by animals $(n=36,20 \%)$, particularly in cattle $(n=23,13 \%)$ (additional file 1). Predominantly, stx $1 a$ and $s t x 2 a$ gene variants are usually identified in human isolates $(n=68 / 93(72 \%)$ and $n=71 / 102(64 \%)$, respectively). Other frequent gene variant in the database, stx2c can be found mostly in animals $(n=21,54 \%)$ followed by humans $(\mathrm{n}=13,33 \%)$ (Fig. 1c). It was confirmed that all STEC genomes had their stx genes in intact prophage regions (except for two in questionable prophages), as classified by PHASTER. This program classifies prophage regions as intact (score above 90), questionable (score between 60 and 90) or incomplete (score less than 60). The score calculation is based on the number of genes related to a known phage and the presence of specific genes (coding proteins involved in phage structure, DNA regulation, insertion, and lysis) [27].

\section{Stx phages can be grouped in four clusters, three subclusters and one singleton}

A dataset of 279 Stx phages were retrieved from the previous group of STEC genomes as of September 2019 (additional file 2) to compare the genomic features of Stx phages. We noted that the genome sizes vary from 31 to $122 \mathrm{~kb}$, containing between 38 and 179 predicted genes. Stx phages were detected in STEC strains with a known in silico determined $\mathrm{O}$ antigen (46 different $\mathrm{O}$ antigens) (additional file 2). Comparative analysis of all Stx phage genomes sorted 24,970 predicted genes into 1, 838 phamilies (phams) of related sequences, of which 
A

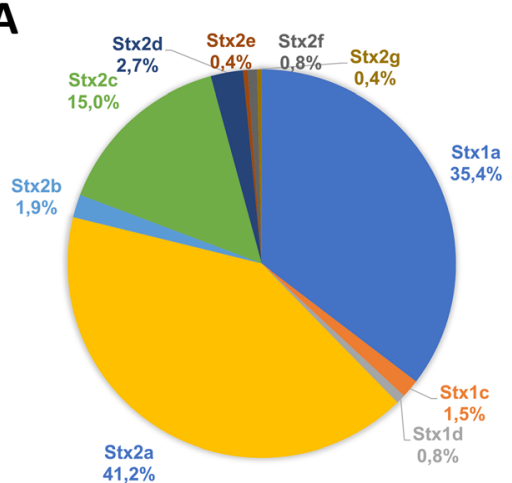

B

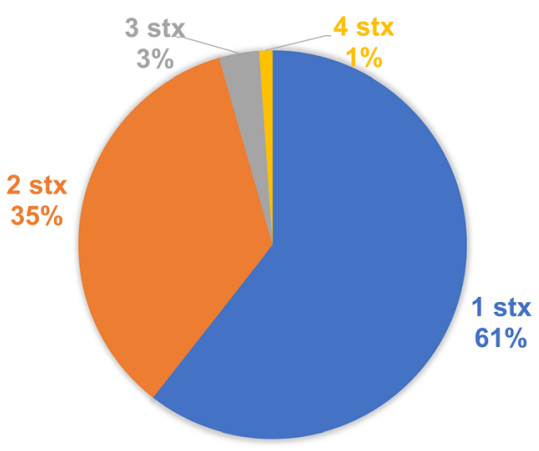

C
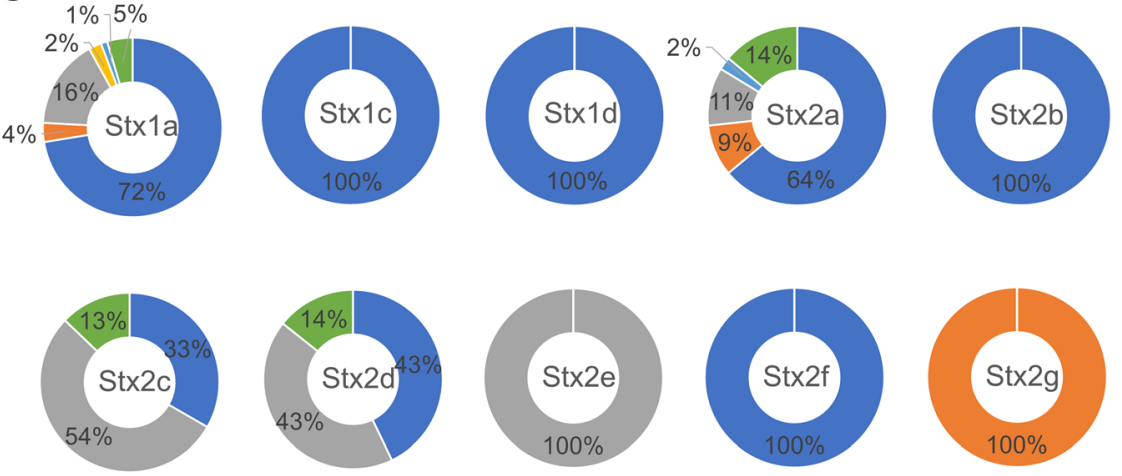

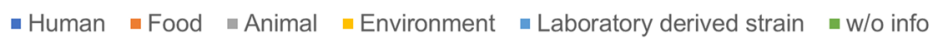

Fig. 1 Distribution of stx genes. a Percentage of each Shiga toxin subtype/variant throughout the E. coli dataset. $\mathbf{b}$ Number of stx genes in a $E$. coli strain. c The sources were sorted into human, food, animal, environment (creek), mutant, and when no information was available as w/o info. Information collected from the GenBank database for each STEC strain

677 possess only a single sequence (orphams) (additional file 3). As expected, the most conserved phams coded for functions related to the Shiga toxin protein which is composed by a single $30 \mathrm{kDa}$ subunit A and a pentamer of $70 \mathrm{kDa}$ subunits B [28]. Shiga subunit A (pham 538) is present in all Stx phages, and Shiga subunit B (pham 1015 ) is missing in only one Stx phage (the gene coding for Shiga subunit $B$ has been deleted on the phage KF030445) (additional file 2). Other conserved phams are related to: a $\mathrm{Rz}$ i-spanin (pham 1301) present in 277 Stx phages, an SASA family carbohydrate esterase (pham 383) present in 258 Stx phages, and a late gene $Q$ regulator (pham 1422) present in 216 Stx phages. Based on the average shared gene content, Stx phage genomes are grouped into four clusters (A to D), three subclusters (A1 to A3) and one singleton (with no close relatives) (Fig. 2 and additional files 5,6, 7, 8, 9, 10, 11). Generally, all genomes are organized into a rightwards-transcribed left arm containing structural genes, a central leftwardsand rightwards-transcribed integration cassette, and a rightwards-transcribed right arm coding for stx genes adjacent to the lysis cassette. The Fig. 3 represents a typical genomic organization of Stx phages. The central block is the most diverse within the clusters.

\section{Cluster A - a diverse group of siphovirus-like phages}

Cluster A is the largest group ( $n=159 / 279,57 \%)$, subdivided into three subclusters (additional file 4). Members of this cluster vary considerably in genome size (31$119 \mathrm{~kb})$, in predicted number of genes (38-179) and in shared gene content (18-100\%, mean of $34 \%)$. Therefore, cluster A is formed on the basis of Stx phage genomes sharing a meaningful (> $35 \%$ ) gene content to at least one member present in cluster A. Most members are predicted siphoviruses, except eight phages for which the virion morphology was impossible to predict (additional file 2). The genomes of cluster A members are organized according to the three major blocks mentioned above. Again, these blocks contain structural genes, integration cassette, and the lysis cassette together with $s t x$ gene (additional file 5, 6, 7). They were found to contain stx 1 genes $(\mathrm{n}=72,45 \%)$, being all of $\operatorname{stx} 1 a$ 
A cluster View

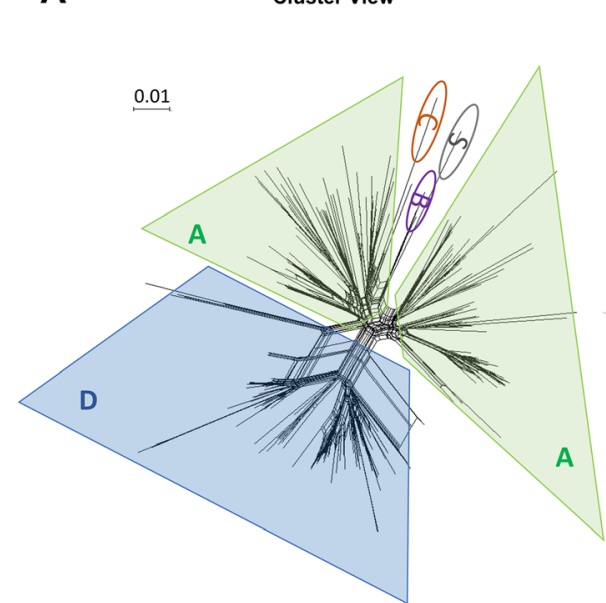

B

Upper view

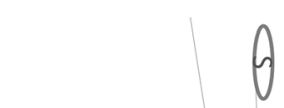

G

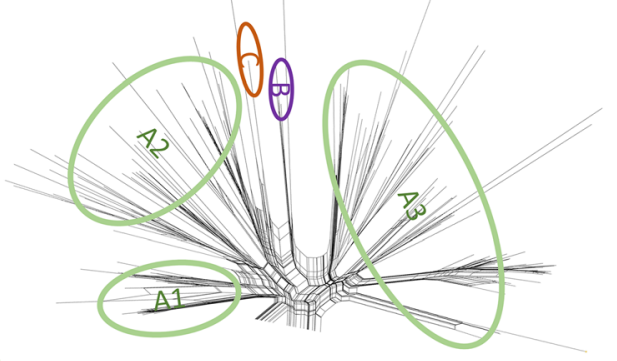

Fig. 2 Diversity of STEC Stx phage genomes. The diversity of the 279 STEC stx phages was analyzed converting the shared proteins (phams) into a distance binary matrix using a python script and visualized in Splitstree (3D representation into 2D space). Clusters (a) and subclusters (b) are divided by colored circles (Green is Cluster A (subcluster A1 to A3), Purple is Cluster B, Orange is Cluster C, Blue is Cluster D, and Grey is

Singleton). The scale bar indicates 0.01 substitution

variants or stx 2 genes $(n=87,55 \%)$, divided into five variants: $\operatorname{stx} 2 a(n=34), \operatorname{stx} 2 b(n=4), \operatorname{st} x 2 c(n=40), \operatorname{st} x 2 d$ $(n=8)$ and $\operatorname{stx} 2 g(n=1)$ (additional file 2).

Subcluster A1 members $(n=22 / 159,14 \%)$ (additional file 5) have a wide pairwise shared gene content (42$100 \%$, mean of $67 \%$ ) (additional file 4 ) and are detected in O111 $(n=12)$, O117 $(n=1)$ and O157 $(n=9)$ strains, isolated from different sources (human, animal and food), in several countries (additional file 2). Two integrase genes were identified, the site-specific integrase (pham 57), and the integrase IntS (pham 1521). In both cases the integrase transcription is leftwards. The position of transposase gene within the genomes is not always the same. All phages carry the same $\mathrm{Q}$ regulator (pham 1422) (additional file 3). Subcluster A2 $(n=50 /$ $159,31 \%$ ) (additional file 6) is the most diverse group, with a wide pairwise shared gene content (15-90\%, mean of $39 \%$ ) (additional 4). Host strains from this group present a great diversity of O-antigens $(n=24)$, and are mostly isolated from human sources $(n=47 / 50$, $94 \%$ ) (additional file 2). Integrases were either of tyrosine-type integrase (phams 1704,1590 or 797 ) or site-specific integrase (phams 57, 23, 1536, 536 or 535) transcribed in both directions. No transposase genes were identified. In this subcluster several genes coding for Q regulator were found (pham 1422, 528, 984, 1414).

Structural genes
Rightwards transcribed module
Lig. $\mathbf{3}$ Common genomic structure of Stx phages. To maintain consistency, all genomes were set to start at their terminase genes. Three general
modules are identified. The first is related with DNA-packaging and morphogenesis (e.g. capsid, tails, packaging genes); The second is composed
by the integration cassette and regulatory genes (e.g. lexA, Cro, $\mathrm{Q}$ antiterminator); The third is composed by the virulence (Shiga toxin $\mathrm{A}$ and $\mathrm{B}$
genes) and the lytic cassette (holin (S), endolysin (R) and i-spainin/O-spanin (Rz/Rzl) genes)


Subcluster A3 $(n=87,55 \%)$ is the largest group of phages within cluster A (additional file 7), with shared genes ranging between 23 and $100 \%$ (mean of $50 \%$ ). The strains $\mathrm{O}$-antigens were also quite diverse $(\mathrm{n}=20)$, with two strains with unidentified O-type (additional data 2). Integrase genes are diverse (site-specific integrase versus tyrosine-type recombinase/integrase) that can either be transcribed leftwards or rightwards. There are phage genomes that carry more than one integrase gene. In this subcluster several genes coding for $\mathrm{Q}$ regulator were found (pham 1422, 978, 984 or 528).

\section{Cluster B - a distinct group of siphovirus-like phages}

Cluster B $(n=3 / 279,1 \%)$ is a small group composed of three predicted siphoviruses (additional file 2) with high shared gene content (72-86\%, mean of $85 \%$ ) (additional file 4). Their genomes are relatively small (39-51 kb), with 61 to 80 predicted genes (additional file 8). All three Stx phages were detected in strains with different O-antigen (O26, O63 and O145) from different sources (pigeons and human). Curiously, cluster B members are the only ones containing the $s t x 2 f$ gene variant (additional file 2). While the beginning and end of the three genomes are similar, the middle module is the most diverse, especially for phage LN997803, as seen by the purple lines (no shading reflets the lack of DNA similarity below the cut-off of $10^{-4}$ of BlastN [29], additional file 8). Interestingly, although they contain several integrases, pham 536 is present in all three phages. As seen in other clusters, there is more than one transposase within each genome.

\section{Cluster C - myovirus-like representatives}

Cluster C $(n=2 / 279,0.72 \%)$ is the smallest group (additional file 9) with a predicted myovirus and an unclassified phage, with relatively low shared gene content $(35 \%)$. The genomes vary in size $(43-78 \mathrm{~kb})$, and therefore vary in the number of predicted genes (66 vs. 114) (additional file 2). Stx phages carry the stx2e gene variant, the only ones found in the dataset. These phages were detected in strains of different O-antigen strains (non-identified and O116), and the source and country of isolation are also different, from a human in Germany and from a pig in China, respectively. The most conserved phams between these phages are the ones responsible for the lysis-lysogeny decision (CI and LexA proteins), Shiga toxin and the lysis cassette. The Q regulators are different (pham 1422 vs. pham 1424).

\section{Cluster D - a big group of close podovirus-like phages}

Cluster D ( $n=114 / 279,41 \%)$ contains a vast number of genomes of predicted podoviruses, with a wide shared gene content (25-100\%, mean of $55 \%$ ) (additional file 4). Some phages have an even lower shared gene content with some members, due to the lack of the first module (phams related with structural proteins) (additional file 10). The genome size ranges from 37 to $122 \mathrm{~kb}$, however, most fall between 60 and $81 \mathrm{~kb}(n=103)$. The number of predicted genes is between 64 and 161 . Phages were detected in several strains of different O-antigens $(n=25)$, and from two strains of Shigella spp. (S. flexneri 2a and $S$. sonnei 75/02 were included as representatives of Stx phages detected in other close related species [30]), being isolated from a wide range of sources and countries. Their genomes have either stx 1 genes $(n=24,21 \%)$, divided into stx 1a $(n=22)$, stx $1 c(n=1)$ and stx $1 d(n=1)$; or $\operatorname{stx} 2(n=$ 88, $77 \%)$, divided into $s t \times 2 a(n=62), s t \times 2 c(n=2), s t \times 2 i$ $(n=1)$. The differentiation of the remaining stx 2 genes was not possible (additional file 2). Phages have either the site-specific integrase transcribed leftwards (phams 57 (the most common), 1536 or 535) or a combination of integrase IntS transcribed leftwards (pham 1521) with site-specific integrases transcribed rightwards (pham 1536). The number of transposases varies considerably, with some genomes having one or more copies transcribed in opposite directions (e.g. phams 238, 154, which seems to appear always in combination). Other phage genomes have only one transposase transcribed leftwards (pham 238) after the stx gene. The Q antiterminator is the most common regulator identified in our dataset (pham 1422). For most members $(n=108)$, the first module is similar (as depicted by the purple lines in map of additional file 10). However, some phages have differences in the tail genes. We noted that within this cluster, there are two podoviruses and one unclassified phage (STX2A_ CP027445.1_2, STX2A_CP027459.1_10, STX2A_ CP013029.1_11) with considerably lower shared gene content (28\%) (additional file 4), that have two blocks with predicted genes for the lytic cassette (additional file 10). This phenomenon is also observed for phages in other clusters.

\section{Singleton - a very singular phage}

The singleton identified (STX1D_CP027447.1_5) (additional file 2) shares fewer than $28 \%$ genes to any of the Stx phage genomes in the dataset. Its genome has a size of $79 \mathrm{~kb}$ with 119 predicted genes, one of them being the $s t x 1 d$. About 48 predicted genes are orphams, being the remaining phams present in cluster A genomes. The genome structure is similar to the other phages (as represented in Fig. 3), having several integrases annotated, but no transposase was identified (additional file 11).

\section{The regulatory region structures for lysis-lysogeny decision are diverse}

Stx phages are known to be lambdoid phages for which several mechanisms for prophage induction have been identified [31]. In our study it was possible to identify a vast number of phams related to the lysis-lysogeny 
regulation, as $\mathrm{Q}$ antiterminator protein [21], LexA regulator [32], antirepressor Ant [33] or Cro/CI repressor proteins [34]. The phams that have a conserved domain are shown in Table 1. As observed in other studies [35], the regulatory region is located upstream of the stx genes and the lysis cassette (additional files 5, 6, 7, 8, 9, $10,11)$. Overall, it seems that phages within the same cluster (or subcluster) share the same organization.

Table 1 Lysis-lysogeny related phams identified in the Stx phage genomes

\begin{tabular}{|c|c|c|c|}
\hline Pham & \# Phage & Function & Domains \\
\hline 78 & 7 & Helix-turn-helix transcriptional regulator (Cro/C1-type HTH and peptidase s24 domains) & COG2932 \\
\hline 87 & 4 & Helix-turn-helix transcriptional regulator $\mathrm{Cro}$ and $\mathrm{Cl}$ & pfam01381 \\
\hline 115 & 3 & Helix-turn-helix transcriptional regulator $\mathrm{Cro}$ and $\mathrm{Cl}$ & pfam01381 \\
\hline 124 & 2 & Repressor LexA & COG1974 \\
\hline 237 & 15 & LexA family transcriptional regulator & pfam01726 \\
\hline 257 & 116 & LexA family transcriptional regulator & COG1974 \\
\hline 393 & 15 & Helix-turn-helix transcriptional regulator (Cro/C1-type HTH and peptidase s24 domains) & COG2932 \\
\hline 507 & 22 & Transcriptional regulator $\mathrm{Cro}$ and $\mathrm{Cl}$ & pfam01381 \\
\hline 528 & 18 & Antitermination protein & pfam03589 \\
\hline 603 & 3 & Helix-turn-helix transcriptional regulator $\mathrm{Cro}$ and $\mathrm{Cl}$ & pfam01381 \\
\hline 629 & 35 & Phage antirepressor Ant & COG3561 \\
\hline 641 & 13 & Regulatory protein Cll & pfam05269 \\
\hline 649 & 89 & Phage antirepressor Ant & COG3561 \\
\hline 837 & 3 & Helix-turn-helix transcriptional regulator $\mathrm{Cro}$ and $\mathrm{Cl}$ & pfam01381 \\
\hline 874 & 1 & Helix-turn-helix transcriptional regulator $\mathrm{Cro}$ and $\mathrm{Cl}$ & pfam01381 \\
\hline 901 & 138 & Hypothetical protein (CII regulatory) & pfam05269 \\
\hline 907 & 5 & Transcriptional regulator Cro superfamily & COG4197 \\
\hline 921 & 22 & Helix-turn-helix transcriptional regulator (Cro/C1-type HTH and peptidase s24 domains) & COG2932 \\
\hline 922 & 9 & HTH-type transcriptional regulator RdgA (Cro/C1-type HTH and peptidase s24 domains) & COG2932 \\
\hline 968 & 1 & Antiterminator Q & pfam06530 \\
\hline 978 & 29 & Antiterminator Q protein & pfam06530 \\
\hline 984 & 13 & DUF1133 family protein (Q antiterminator HHPred) & cl29970 \\
\hline 1009 & 8 & Cro/Cl family transcriptional regulator & COG4197 \\
\hline 1010 & 1 & Rha family transcriptional regulator Cro superfamily & COG4197 \\
\hline 1059 & 7 & LexA family transcriptional repressor & COG1974 \\
\hline 1199 & 1 & Transcriptional regulator lambda repressor-like DNA-binding domain & COG3423 \\
\hline 1204 & 2 & LexA family transcriptional regulator & COG1974 \\
\hline 1204 & 2 & LexA family transcriptional regulator & COG1974 \\
\hline 1318 & 11 & Helix-turn-helix transcriptional regulator $\mathrm{Cro}$ and $\mathrm{Cl}$ & pfam01381 \\
\hline 1392 & 1 & Helix-turn-helix transcriptional regulator $\mathrm{Cro}$ and $\mathrm{Cl}$ & pfam01381 \\
\hline 1422 & 216 & Late gene regulator $\mathrm{Q}$ & pfam06530 \\
\hline 1424 & 7 & Antitermination protein & pfam06530 \\
\hline 1457 & 2 & LexA family transcriptional regulator & COG1974 \\
\hline 1550 & 1 & Antiterminator Q & pfam06323 \\
\hline 1642 & 2 & Transcriptional regulator Cro protein & pfam14549 \\
\hline 1673 & 3 & Helix-turn-helix transcriptional regulator $\mathrm{Cro}$ and $\mathrm{Cl}$ & pfam01381 \\
\hline 1763 & 64 & Helix-turn-helix transcriptional regulator (Cro/C1-type HTH and peptidase s24 domains) & COG2932 \\
\hline 1266 & 1 & Phage antirepressor Ant & pfam03374 \\
\hline 1639 & 16 & Putative antirepressor & pfam03374 \\
\hline 1668 & 1 & Phage antirepressor Ant & COG3561 \\
\hline
\end{tabular}


The $\mathrm{Q}$ regulator is an important member of the regulatory region of Stx phage genomes [21]. Indeed, several phams were annotated with this function, being the most conserved: pham 1422 (present in 216 phages of multiple clusters), pham 978 (present in 29 siphoviruses), and the pham 528 (present in 18 siphoviruses and unclassified phages). Another known gene of this region is the repressor $\operatorname{lex} A$, for which there are six phams identified, all with the same domain (COG1974). The most common is pham 257 (present in 116 phages of all clusters), followed by pham 237 (present in 15 siphovirus and unclassified phages of cluster A). Unexpectedly, for some phages, this regulator was not detected. LexA is normally transcribed leftwards and present once in the genome (additional file 3). Nevertheless, few phages have two different phams related to LexA functions (phams 1059 and 237). Repressors Cro and CI are important members that regulate the lytic excision of the prophage [36] by self-regulating its promoter, inhibiting the expression of the all others genes of the prophage [33]. Several phams $(n=18)$ with these regulatory properties were identified (Table 1), usually transcribed rightwards as the Q antiterminator phams (Table 1, additional file 3). The antirepressor Ant is an important gene of the superinfection ability of P22 phage [33], being present in 126 Stx phage genomes (phams 649 and 629 in 89 and 35 members, respectively) (Table 1).

\section{The conserved lysis cassette is located upstream the stx genes}

The prototype phage Lambda genome is known to have a lysis cassette composed of the $S$ (holin), $R$ (canonical endolysin) and $R z / R z 1$ (i-spanin/o-spanin, being the latter embedded in the +1 reading frame of $R z)$ genes [37]. In our dataset, the endolysin gene is conserved (phams 858, 838 and 762, shared by 225, 44 and two phages, respectively). Both phams 858 and 838 are predicted SAR (signal-arrest-release) endolysins having an $\mathrm{N}$-terminal R21-like domain and present in all clusters (except cluster $\mathrm{C}$ ). On the other hand, pham 762 is predicted to be a canonical endolysin, having a metallopeptidase domain without a signal peptide, and exclusively present in cluster $\mathrm{C}$ members. The holin function is split in more genes (phams 52, 108, 135, 374, 891 and 1132, in which the latter two are the most conserved, present in 138 and 72 phages, respectively). Conceivably, this protein functions as a pin-holin (in phams 108, 135, 374, 891 and 1132) and is associated with a predicted SAR endolysin or acts as a classical holin (in pham 52) when associated with canonical endolysins (exclusively found in cluster C). The $R z / R z 1$ (pham 1301) is one of the most conserved genes among the genomes of the prophages analyzed (present in 277 phages), only surpassed by the genes coding for Shiga toxin subunits A and B (pham 538 and pham 1015, respectively), present in all phages. The embedded $R z 1$ o-spanin is not represented in the genomics maps due to the automatic annotation of the phage genomes. The lysis cassette map of some phages is represented in additional file 12. The holin was not found in 11 Stx phages, of which 10 phages (from subcluster A1) only have the i-spanin (pham 1301) identified (with no endolysin or holin detected) (Additional file 5)

\section{Discussion}

This study compared 787 complete E. coli genomes available at GenBank database as of September 2019. Their genomes were typed for their antigens $(\mathrm{O}$ and $\mathrm{H}$ antigens), using the Web tool SerotypeFinder [38], and stx genes, using the Web tool VirulenceFinder [39]. Additionally, a dataset of 279 Stx phages was curated and their genomes grouped into clusters based on gene content similarity. The dataset was constructed through PHASTER, an online tool able to predict prophage regions within a bacterial genome [40]. Additionally, 55 Stx phage genomes (including two of Shigella spp., reported to be similar do STEC Stx phages [41, 42]) were directly retrieved from the GenBank and added to our dataset.

For several years, STEC strains were classified using a simple scheme of $\mathrm{O} 157$ and non-O157 [43], as this STEC serotype has been considered the most pathogenic, making it the most extensively studied in reference laboratories worldwide [44]. Recently, other serotypes have also been recognized as important pathogens, as reflected in STEC detection standards used nowadays (the technical specification of the International Organization for Standardization ISO/TS 13, 136:2012 and Microbiology Laboratory Guidebook 5 C.00 from United States Department of Agriculture). Indeed, we found the O157:H7 to be the most represented serotype (26.8\%); but others, such as O104:H4 and O26:H11, were also highly represented in our dataset (both around $7 \%$ ), which can be explained by recent outbreaks where new STEC serotypes have been continually emerging [14, 45]. Regarding the Shiga toxins, three subtypes for Stx1 (a, c, and d) and nine for Stx2 (a to i) are currently known [3-6]. Different studies have shown that Stx2a is the most virulent, being often associated with HUS. However, Stx1a, Stx2c or Stx2d toxins are also associated with the development of HUS [46]. As expected, the subtype more detected in humans was the Stx2a, either alone or associated with Stx1a or Stx2c (additional file 1). Several subtypes, namely Stx2e-g, were less represented (Fig. 1a), which can be explained by their inherent low virulence, and therefore rarely detected. In fact, most strains $(63 \%)$ included in our 
dataset were isolated from humans, of which $21 \%$ were from confirmed illness cases (additional file 1).

We found all stx genes within intact Stx phages (except for two questionable prophages), rendering them the ability to potentially excise and infect new hosts, as observed in Shigella and Aeromonas strains, which carry Stx phages homologous to the ones detected in STEC [41, 47]. We also found a significant portion (39\%) of STEC strains with more than one Stx phage (Fig. 1b). Fogg et al. (2011), demonstrated that phage $\Phi 24_{\mathrm{B}}$ could insert itself multiple times on the same host [33]. The integrase, outside of the regulatory control of phage repression region, could be the responsible for this behavior. As the integrase is constantly expressed, this would allow the prophages to be established. Similarly, in our study, multiple integrases transcribed outside the regulatory region were also observed, e.g. Subcluster A1 (additional file 5), which could also explain multiple infection events.

These Stx phages' ability to infect distinct serotypes, even from different pathotypes, can lead to serious global health outcomes. A good example are the Stx2aconverting phages infecting E. coli O104:H4, a known Enteroaggregative E. coli (EAEC) responsible for the outbreak of 2011 in Germany and other European countries $[45,48]$, which became known as a novel hybrid pathotype (EAEC/STEC) [48]. In our dataset, 14 predicted podoviruses were similar, sharing $88 \%$ of its genes to the Stx2a-converting phage isolated during the 2011 outbreak (additional files 2, 4 and 10). Moreover, a similar high shared gene content between 81 and $100 \%$ is observed to others prophages detected in different serotypes, as $\mathrm{O} 2: \mathrm{H} 27$ isolated from cattle [49], O111:H8 isolated from human [50] and O168: $\mathrm{H} 8$, isolated from food [51]. This suggests the ability of some prophages to exchange hosts with different serotypes. Beutin et al. hypothesis that STEC strains not commonly associated with human disease can work as the source of Stx2aconverting phages, able to convert EAEC into a high virulent strains [49], is in agreement with our analysis that identified 13 EAEC/STEC strains.

Through multiple lines of evidence, the STEC Stx phages were shown to be extremely diverse. First, their genome sizes ranged between 30 and $122 \mathrm{~kb}$, broader than previously anticipated in 2015 (30$70 \mathrm{~kb}$ ) [17]. Of note, some STEC genomes appeared to have prophages inserted in series, which can be an artefact from PHASTER that failed at recognizing the genomes ends of prophages in close proximity. The apparent increased size, comparatively to the archetype phage lambda $(45.5 \mathrm{~kb})$, suggests that the additional genes acquired serves to modulate their lysogens rather than being involved in the phage core functions per se. Some phams identified in these longer genomes, are related to metabolism, such as D-serine ammonia-lyase (pham 184), Exodeoxyribonuclease VIII (pham 1056) and Multidrug efflux MFS transporter permease subunit EmrY (pham 1610), being inserted in the middle module. Second, the genomes could be grouped into four clusters and one singleton based on shared gene content similarity, although even within the clusters, a vast range of shared gene values was observed.

Interestingly, cluster assignment is in agreement with their predicted virion morphologies, i.e. Siphoviridae (clusters A-B), Myoviridae (cluster C) and Podoviridae (cluster D). The fact that phage genomes from clusters $B$ and $C$ exclusively code rare (or underestimated) toxins (stx $2 f$ and $s t x 2 e$, respectively) from a pool of 12 detected variants, is a significant trend that warrant further investigation. Third, besides generally being organized into three major modules coding for morphogenetic (rightwardstranscribed); integration cassette (transcribed both ways) and lysis cassette functions (rightwards-transcribed), the genomes have frequent small non-homologous modules and synteny breaks between genomes, similar to what was observed in phage genomes infecting other hosts (e.g. other Enterobacteriaceae, Staphylococcus, Pseudomonas, Mycobacterium) [23, 25, 26, 52]. This high mosaicism pattern can be interpreted as a continuous adaptation of the phage fitness to infect other hosts and/or to survive in several environments likely driven by HGT. Finally, the vast diversity of Stx phages is also seen at the gene level. From a total of 20,382 predicted genes, 1,838 families could be sorted using Phamerator. Most (78\%) were shared by ten or fewer Stx phages, with a significant percentage (37\%) being unique genes (displayed in genome maps as white boxes). The fact that this percentage of single gene lies in close proximity of other phage populations infecting hosts with higher taxonomic levels, yet similar population sizes (e.g. Staphylococcus phages have $35 \%$ single gene), is very meaningful. This vast gene diversity is likely driven by continuous gene influx from novel bacterial hosts and/or other phages by HGT and demonstrates an untapped reservoir of genes with potential ecological (e.g. repressor, toxins) and biotechnological (e.g. lytic proteins, recombinases) roles.

Several mechanisms can be responsible for the incorporating genes by prophages, including transposases [52], which are the most abundant genes in nature [53]. The transposase and insertion sequences (IS) are commonly found in bacterial genomes, being responsible for several gene arrangements [54], which modulate the genome evolution [55]. Several transposases and IS elements were identified in our study, including within the same Stx phage genome. Some were found near stx genes, either up or downstream. Nevertheless, most transposases were found on the morphogenetic and integration 
modules (additional files 5, 6, 7, 8, 9, 10, 11). Such mobile elements' insertion is an important evolutionary element and diversification of the lysogens, since it can introduce new genes into the genomes [56]. It can also result in the regulation of the adjacent genes. Kusumoto et al., in 2000, associated the regulation of Shiga toxin productivity with the integration of an IS1203v element (classified into the IS3 family) adjacent to the stx2 genes. It was demonstrated that with the IS element's excision, the ability to express Shiga toxin was regained [54]. As seen in our pham dataset, the IS3 family is the most distributed in STEC Stx phages, present in 109 genomes (additional file 3). Further investigation is needed to fully understand the impact of the insertion and excision of these elements on the evolution, regulation, and perhaps (in)activation of Stx phages.

It is generally accepted that the Shiga toxin production and the transference of $s t x$ genes are related to the induction and subsequent excision of Stx phages [57]. The phams related to the lysis-lysogeny decision were common for all Stx phages of the dataset (additional file 3 and Table 1), as reported in other Stx phage populations [5860]. However, several organizations were detected, with different additional genes incorporated adjacent to Cro, $\mathrm{CI}$ and Q regulators (additional file 5, 6, 7, 8, 9, 10, 11). The diversity in the regulatory genes' architecture can explain distinct Shiga toxin expression levels, as well as the strains' potential to produce phage particles without inducer agents [61]. In fact, spontaneous production of Shiga toxin was previously observed for phage 933W [35]. In opposite to phage Lambda, phage 933W does not form a DNA loop, responsible for connecting adjacent operators, repressing promotors related to the lytic growth. In Bullwinkle et al., the authors described that the concentration of repressor needed to activate or repress the lytic functions is low, explaining in part the spontaneous phage induction. Therefore, phage 933W has evolved to regulate its lysogen through different strategies [34]. To better understand the extent of the different regulatory mechanisms in Stx phages, more in-depth studies are needed. The location of virulence genes downstream of lysis module is common, associating the virulence feature's expression to phage induction [25]. An important aspect of lambdoid phages is that the integrase genes are regulated by the lysis-lysogeny regulatory region [62]. However, it was reported that, for some Stx phages, these genes are transcribed in opposite directions. This leads to a constant expression of integrases allowing multiples prophages in the same lysogen [33]. In fact, several integrase phams were identified in our database, transcribed in both directions, usually outside the lysis-lysogeny regulatory region.

Regarding with the lytic cassette, that of phage lambda contains a canonical holin that forms large pores exposing the peptidoglycan locally to endolysins in a genetically determined time [63]. However, almost all Stx phages herein analyzed (99\%) have a predicted SAR endolysin and pin-holin. The SAR endolysin $\mathrm{N}$-terminal signal is known to mediate export through the host secretory system, being activated by membrane depolarization performed with the pin-holins, which is believed to make small holes in the host cell membrane [63]. We found the lysis genes to be in close proximity with each other and located downstream the integration cassette and adjacent to the $s t x$ genes. In-between the stx and the lysis genes, there is a region, typically around 3 to 3.5$\mathrm{kb}$, where several genes are found, which could also have impact their lysogens, therefore warranting further investigation. This hypothesis of having additional virulence genes located downstream the integration cassette would mirror the generic organization of other prophages that infect different hosts (e.g. Staphylococcus) [25]. All STEC Stx phage late genes are located downstream and in the same transcriptional orientation of the anti-terminator gene Q (with fewer phams being transcribed in the opposite direction), controlling their expression [64, 65]. We were also able to demonstrate that phage genomes carrying different stx alleles share similar Q genes. However, in some cases, the Q gene was incomplete or missing, presumably due to recombination events [66]. The synthesis of $s t x$ genes is then unequivocally linked to the cell lysis once the STEC Stx phages enter the lytic cycle, as experimentally validated in several studies [67-69]. Nevertheless, there is still a good evolutionary theory to be disclosed to support the discrete location of the virulence factors in Stx phage genomes, as other locations within the late region would serve the same goal.

\section{Conclusions}

There is a considerable diversity of STEC serotypes that do not fall into the well-known O157:H7 and the so-called top six non-O157 serotypes (O26, O45, O103, O111, O121, O145), which carry complete Stx phages with stx genes variants. All stx genes in STEC strain genomes seem to be phage-borne located in intact prophages, regardless of the phage type, STEC serotype, geographical region, and sample origin. Stx phages were divided into four clusters and one singleton, based on their gene shared content, which is in agreement with their predicted morphologies. Despite the vast regulatory region structures for lysis-lysogeny, the conserved stx location in the lytic cassette, strongly suggests a role of $s t x$ expression during prophage excision, but other alterative mechanisms cannot be discarded without further investigation.

\section{Methods}

The $E$. coli prophage dataset was constructed using complete $E$. coli sequenced genomes $(n=787)$ deposited at GenBank in September 2019. In silico typing (O and 
$\mathrm{H}$ antigens) was performed using SerotypeFinder 2.0 (additional file 1) [38], using $85 \%$ as threshold for identity and a minimum length (number of nucleotides a sequence will overlap) of $60 \%$.

Biopython 32 package was used within the conda environment, and python scripts were used to automatically scan each $E$. coli genomes for prophages through PHASTER API [40]. Prophages were retrieved and identified as incomplete $(n=1757)$, questionable $(n=763)$ or intact $(n=4392)$, depending on the completeness or their potential viability. Each prophage was screened for stx genes (stx $1 a, s t x 1 c-d, s t x 2 a-g$, for both subunits A and B) using VirulenceFinder, with standard parameters [70], resulting in 259 intact Stx phages (for incomplete prophages no stx genes were found; moreover, only two questionable phages were detected that were not included on the final dataset). The final dataset of 279 Stx phages was constructed using 224 randomly selected intact Stx phages from the previous analysis with $55 \mathrm{Stx}$ phages directly retrieved from GenBank (including two phages from Shigella spp. since these Stx phages are also detected in foodborne pathogens [71]) (additional file 2).

For consistency, all Stx phage genomes (from PHAS TER or GenBank) were set to start at the terminase genes, and re-annotated using Geneious Prime [72]. Protein functions were manually inspected using BLASTP (blast.ncbi.nlm.nih.gov) against the NCBI nonredundant protein database and the NCBI Conserved Domain Database (CDD) with CD-Search (ncbi.nlm.nih.gov/Structure/cdd). In some cases, they were inferred based on structural similarity using HHpred server with Protein Data Bank database (toolkit.tuebingen.mpg.de/ \#/tools/hhpred). An E-value cutoff of $1 \times 10^{-5}$ were used for all searches.

Whole-genome comparisons of Stx phages were made using Phamerator [73], which allowed the analysis of the shared gene content by grouping genes into phams with Kclust algorithm (additional files 3-4) and to generate comparative genome maps using the "Align Two Sequences" algorithm of BLASTN (additional files 5, 6, $7,8,9,10,11)$. The shared gene content was visualized in SplitsTree [74]. Phage genomes were assigned into only one cluster when sharing $35 \%$ of shared genes (phams) or as singletons if sharing fewer genes to all members, a metric previously used to assign phage membership [25]. Stx phage morphology was predicted based on the most homolog phage found using BLASTN (blast.ncbi.nlm.nih.gov) [75] using "Tailed phages" (taxid:28,883) database.

\section{Abbreviations}

STEC: Shiga toxin-producing Escherichia coli; stx: Shiga toxin;

phams: Phamilies; HUS: Hemolytic-uremic syndrome; Stx1: Shiga toxin subtype 1; Stx2: Shiga toxin subtype 2; HGT: Horizontal gene transfer;

S: Holin; R: Canonical endolysin; Rz/Rz1: i-spanin/o-spanin, being the latter embedded in the +1 reading frame of Rz; SAR: Signal-arrest-release; EAEC: Enteroaggregative E. coli; IS: Insertion sequence

\section{Supplementary Information}

The online version contains supplementary material available at https://doi. org/10.1186/s12864-021-07685-0.

Additional file 1. E. coli strains information. Serotype and Virulence tab contains the information of all 787 strains, while the Stx carriers tab has only the strains where at least one stx gene is found. For all strains, the $O$ and $\mathrm{H}$ antigens, stx genes, origin (source of isolation) and country of isolation is provided, using SerotypeFinder, information retrieved from GenBank or from a reference article (when available). w/o, information not available.

Additional file 2. STEC Stx phages information. The information for all 279 Stx phages used in the final dataset is provided. Phages were characterized for: sequence length, number of proteins, cluster, predicted family, similar phage (hit of BlastN, query cover, E-value, percentage identity), Stx subtype/variant, the lysogen O-type, as well as origin (source of isolation) and country of isolation. For those retrieved directly from GenBank database, the reference article is provided. w/o, information not available.

Additional file 3. Phams identified in the Stx phages dataset. The dataset includes 279 Stx phages, encoding a total of 24,970 predicted proteins sorted into 1,838 phamilies (phams) of related proteins, 677 of which were identified as orphans (genes without related sequences) using Phamerator.

Additional file 4. Shared gene content matrix. Phamerator output (1,838 phams) was converted to a matrix of shared gene content. Heat map was created in excel.

Additional file 5. Whole-genome map of subcluster A1 phages. Maps were generated with Phamerator, where pairwise sequence similarity (minimal BLASTN cut-off E value is 10-4) is given according to color spectrum (purple lines for highest and red lines for the lowest nucleotide similarity, no shading shows no similarity with a BLASTN score of 10-4 or better). Ruler corresponds to genome base pairs. Labelled ORFs with predicted function are shown as colored boxes (white boxes represent orphans, singe genes) position above (rightwards transcribed) or below (leftwards transcribed) the bar. Gene numbering reflects the reorganization of genomes. All genomes were set to start at the terminase genes.

Additional file 6. Whole-genome map of subcluster A2 phages. Represented as in Additional file 5.

Additional file 7. Whole-genome map of subcluster A3 phages. Represented as in Additional file 5.

Additional file 8. Whole-genome map of cluster B phages. Represented as in Additional file 5.

Additional file 9. Whole-genome map of cluster $\mathrm{C}$ phages. Represented as in Additional file 5.

Additional file 10. Whole-genome map of cluster D phages. Represented as in Additional file 5.

Additional file 11. Whole-genome map of singleton. Represented as in Additional file 5 .

Additional file 12. Lysis cassette map. The map was constructed using Phamerator using six randomly Stx phages as an example. Genes are labelled with their putative function, and phams with same predicted functional are represented by same color. Similarity is given by purple lines (minimal BLASTN cut-off E value is 10-4).

\section{Acknowledgements}

Not applicable.

\section{Authors' contributions}

GP assisted with experimental design, interpreted the results, and drafted the manuscript. MS performed the bioinformatics work and revised the manuscript. OD revised the bioinformatics work and manuscript. CA and JA 
assisted with experimental design, interpretation of results and edited the manuscript. $\mathrm{HO}$ performed the experimental design, interpretation of results, and help drafted the manuscript. All authors have read and approved the final manuscript.

\section{Funding}

This study was supported by the Portuguese Foundation for Science and Technology (FCT) under the scope of the strategic funding of UIDB/04469/ 2020 unit and the project PhageSTEC PTDC/CVT-CVT/29628/2017 [POCl-010145-FEDER-029628] funded by FEDER through COMPETE2020 (Programa Operacional Competitividade e Internacionalização) and by National Funds thought FCT. GP is recipient of a FCT PhD grant with the reference SFRH/ BD/117365/2016. The funding body had no role in the design of the study and collection, analysis, and interpretation of data and in writing the manuscript.

\section{Availability of data and materials}

Data generated and analyzed throughout this study are included in this published article and in the additional information files. Escherichia coli complete genomes were retrieved from GenBank database and each accession number can be found in Additional file 1. Prophage' genomes were retrieved using PHASTER web tool using the E. coli genomes' accession numbers and can be provided upon request

\section{Declarations}

\section{Ethics approval and consent to participate}

Not applicable.

\section{Consent for publication}

Not applicable.

\section{Competing interests}

The authors declare that they have no competing interests.

\section{Author details}

${ }^{1}$ CEB - Centre of Biological Engineering, University of Minho, 4710-057 Braga, Portugal. ${ }^{2}$ INIAV, IP-National Institute for Agrarian and Veterinary Research, Rua dos Lagidos, Lugar da Madalena, Vairão, Vila do Conde, Portugal.

\section{Received: 20 October 2020 Accepted: 7 May 2021}

\section{Published online: 19 May 2021}

\section{References}

1. Cowley LA, Dallman TJ, Jenkins C, Sheppard SK. Phage Predation Shapes the Population Structure of Shiga-Toxigenic Escherichia coli 0157:H7 in the UK: An Evolutionary Perspective. Front Genet. 2019;10(August):1-7.

2. Control EC for DP and. Shiga-toxin/verocytotoxin-producing Escherichia coli (STECNTEC) infection. ECDC Annu Epidemiol Rep 2018. 2020;(April).

3. Scotland SM, Smith HR, Rowe B. Two Distinct Toxins Active on Vero Cells From Escherichia Coli 0157. Lancet. 1985;326(8460):885-6.

4. Scheutz F, Teel LD, Beutin L, Piérard D, Buvens G, Karch H, et al. Multicenter evaluation of a sequence-based protocol for subtyping Shiga toxins and standardizing Stx nomenclature. J Clin Microbiol. 2012;50(9):2951-63.

5. Bai X, Fu S, Zhang J, Fan R, Xu Y, Sun H, et al. Identification and pathogenomic analysis of an Escherichia coli strain producing a novel Shiga toxin 2 subtype. Sci Rep [Internet]. 2018;8(1):1-11. Available from: https:// doi.org/10.1038/s41598-018-25233-x.

6. GROUP FSE, The. Hazard Identification and Characterization: Criteria for Categorizing Shiga Toxin-Producing Escherichia coli on a Risk Basist. J Food Prot [Internet]. 2019 Jan 1;82(1):7-21. Available from: https://meridian.a Ilenpress.com/jfp/article/82/1/7/174565/Hazard-Identification-and-Chara cterization.

7. Orth D, Grif K, Khan AB, Naim A, Dierich MP, Würzner R. The Shiga toxin genotype rather than the amount of Shiga toxin or the cytotoxicity of Shiga toxin in vitro correlates with the appearance of the hemolytic uremic syndrome. Diagn Microbiol Infect Dis. 2007;59(3):235-42.

8. Persson S, Olsen KEP, Ethelberg S, Scheutz F. Subtyping method for Escherichia coli Shiga toxin (Verocytotoxin) 2 variants and correlations to clinical manifestations. J Clin Microbiol. 2007;45(6):2020-4.
9. Shringi S, Schmidt C, Katherine K, Brayton KA, Hancock DD, Besser TE. Carriage of stx2a Differentiates Clinical and Bovine-Biased Strains of Escherichia coli 0157. PLoS One. 2012;7(12).

10. Kawano K, Okada M, Haga T, Maeda K, Goto Y. Relationship between pathogenicity for humans and stx genotype in Shiga toxin-producing Escherichia coli serotype 0157. Eur J Clin Microbiol Infect Dis. 2008;27(3): 227-32.

11. FAO/WHO. Shiga toxin-producing Escherichia coli (STEC) and food: attribution, characterization, and monitoring. Microbiological Risk Assessment Series. [Internet]. 2018. 152 p. Available from: http://www.fao. org/3/ca0032en/CA0032EN.pdf.

12. Caprioli A, Morabito S, Brugere H, Oswald E. Enterohaemorrhagic Escherichia coli: emerging issues on virulence and modes of transmission. Vet Res [Internet]. 2005 May;36(3):289-311. Available from: http://www.edpsciences. orghttps://doi.org/10.1051/vetres:2005002.

13. MATHUSA EC, CHEN Y, ENACHE E, HONTZ L. Non-O157 Shiga ToxinProducing Escherichia coli in Foods. J Food Prot [Internet]. 2010 Sep 1;73(9): 1721-36. Available from: https://meridian.allenpress.com/jfp/article/73/9/1 721/173666/NonO157-Shiga-ToxinProducing-Escherichia-coli-in.

14. Bonanno L, Loukiadis E, Mariani-Kurkdjian P, Oswald E, Garnier L, Michel V, et al. Diversity of shiga toxin-producing Escherichia coli (STEC) O26: H11 strains examined via stx subtypes and insertion sites of Stx and EspK bacteriophages. Appl Environ Microbiol. 2015;81(11):3712-21.

15. Cornick NA, Helgerson AF, Mai V, Ritchie JM, Acheson DWK. In vivo transduction of an Stx-encoding phage in ruminants. Appl Environ Microbiol. 2006;72(7):5086-8.

16. Khalil RKS, Skinner C, Patfield S, He X. Phage-mediated Shiga toxin (Stx) horizontal gene transfer and expression in non-Shiga toxigenic Enterobacter and Escherichia coli strains. Pathog Dis. 2016;74(5):1-11.

17. Krüger A, Lucchesi PMA. Shiga toxins and stx phages: highly diverse entities. Microbiology. 2015;161(2015):451-62.

18. Bonanno L, Petit MA, Loukiadis E, Michel V, Auvray F. Heterogeneity in induction level, infection ability, and morphology of Shiga toxinencoding phages (Stx phages) from dairy and human Shiga toxin-producing Escherichia coli O26:H11 isolates. Appl Environ Microbiol. 2016:82(7):2177-86.

19. Mora A, Blanco M, Blanco E, Alonso MP, Dhabi G, Thomson-carter F, et al. Phage Types and Genotypes of Shiga Toxin-Producing Escherichia coli 0157: H7 Isolates from Humans and Animals in Spain : Identification and Characterization of Two Predominating Phage Types. Society. 2004;42(9): 4007-15.

20. Yara DA, Greig DR, Gally DL, Dallman TJ, Jenkins C. Comparison of Shiga toxin-encoding bacteriophages in highly pathogenic strains of shiga toxinproducing Escherichia coli 0157:H7 in the UK. Microb Genomics. 2020;6(3).

21. Steyert SR, Sahl JW, Fraser CM, Teel LD, Scheutz F, Rasko DA. Comparative Genomics and stx Phage Characterization of LEE-Negative Shiga ToxinProducing Escherichia coli. Front Cell Infect Microbiol [Internet]. 2012; 2(November):1-18. Available from: http://journal.frontiersin.org/article/ https://doi.org/10.3389/fcimb.2012.00133/abstract.

22. Ogura Y, Mondal SI, Islam MR, Mako T, Arisawa K, Katsura K, et al. The Shiga toxin 2 production level in enterohemorrhagic Escherichia coli $0157: \mathrm{H} 7$ is correlated with the subtypes of toxin-encoding phage. Sci Rep [Internet]. 2015;5(October):1-11. Available from: https://doi.org/10.1038/srep16663.

23. Pope $\mathrm{WH}$, Jacobs-Sera D, Russell DA, Peebles CL, Al-Atrache Z, Alcoser TA, et al Expanding the Diversity of Mycobacteriophages: Insights into Genome Architecture and Evolution. Aziz R, editor. PLoS One [Internet]. 2011 Jan 27; 6(1):e16329. Available from: https://doi.org/10.1371/journal.pone.0016329.

24. Grose JH, Jensen GL, Burnett SH, Breakwell DP. Correction: genomic comparison of 93 Bacillus phages reveals 12 clusters, 14 singletons and remarkable diversity. BMC Genomics [Internet]. 2014;15(1):1184. Available from: http://bmcgenomics.biomedcentral.com/articles/https://doi.org/10.11 86/1471-2164-15-1184

25. Oliveira H, Sampaio M, Melo LDR, Dias O, Pope WH, Hatfull GF, et al. Staphylococci phages display vast genomic diversity and evolutionary relationships. BMC Genomics [Internet]. 2019 Dec 9;20(1):357. Available from: https://bmcgenomics.biomedcentral.com/articles/https://doi.org/10.1186/s12 864-019-5647-8

26. Grose JH, Casjens SR. Understanding the enormous diversity of bacteriophages: The tailed phages that infect the bacterial family Enterobacteriaceae. Virology [Internet]. 2014 Sep 19 [cited 2015 Jan 2]; 468-470 C:421-43. Available from: http://www.ncbi.nlm.nih.gov/ pubmed/25240328. 
27. Zhou Y, Liang Y, Lynch KH, Dennis JJ, Wishart DS. PHAST: A Fast Phage Search Tool. Nucleic Acids Res. 2011;39(SUPPL. 2):347-52.

28. Obrig TG. Escherichia coli shiga toxin mechanisms of action in renal disease. Toxins (Basel). 2010;2(12):2769-94.

29. Cresawn SG, Bogel M, Day N, Jacobs-Sera D, Hendrix RW, Hatfull GF. Phamerator: A bioinformatic tool for comparative bacteriophage genomics. BMC Bioinformatics. 2011;12(October).

30. Karmali MA. Factors in the emergence of serious human infections associated with highly pathogenic strains of shiga toxin-producing Escherichia coli. Int J Med Microbiol [Internet]. 2018 Dec;308(8):1067-72. Available from: https://linkinghub.elsevier.com/retrieve/pii/S14384221183 02315.

31. Chakraborty D, Clark E, Mauro SA, Koudelka GB. Molecular Mechanisms Governing "Hair-Trigger" Induction of Shiga Toxin-Encoding Prophages. Viruses [lnternet]. 2018 Apr 29;10(5):228. Available from: http://www.mdpi. com/1999-4915/10/5/228.

32. Nassar FJ, Rahal EA, Sabra A, Matar GM. Effects of Subinhibitory Concentrations of Antimicrobial Agents on Escherichia coli O157:H7 Shiga Toxin Release and Role of the SOS Response. Foodborne Pathog Dis [Internet]. 2013;10(9):805-12. Available from: http://online.liebertpub.com/ doi/abs/https://doi.org/10.1089/fpd.2013.1510.

33. Fogg PCM, Rigden DJ, Saunders JR, McCarthy AJ, Allison HE. Characterization of the relationship between integrase, excisionase and antirepressor activities associated with a superinfecting Shiga toxin encoding bacteriophage. Nucleic Acids Res. 2011;39(6):2116-29.

34. Bullwinkle TJ, Koudelka GB. The lysis-lysogeny decision of bacteriophage 933 W: A 933 W repressor-mediated long-distance loop has no role in regulating 933 W PRM activity. J Bacteriol. 2011;193(13):3313-23.

35. Plunkett G, Rose DJ, Durfee TJ, Blattner FR. Sequence of Shiga toxin 2 phage 933 W from Escherichia coli O157:H7: Shiga toxin as a phage lategene product. J Bacteriol [Internet]. 1999 Mar;181(6):1767-78. Available from: http://www.ncbi.nlm.nih.gov/pubmed/10074068.

36. Hernandez-Doria JD, Sperandio V. Bacteriophage Transcription Factor Cro Regulates Virulence Gene Expression in Enterohemorrhagic Escherichia coli. Cell Host Microbe [Internet]. 2018;23(5):607-617.e6. Available from: https:// doi.org/10.1016/.j.chom.2018.04.007.

37. Summer EJ, Berry J, Tran TAT, Niu L, Struck DK, Young R. Rz/Rz1 Lysis Gene Equivalents in Phages of Gram-negative Hosts. J Mol Biol. 2007;373(5):1098-112.

38. Joensen KG, Tetzschner AMMM, Iguchi A, Aarestrup FM, Scheutz F. Rapid and easy in silico serotyping of Escherichia coli isolates by use of wholegenome sequencing data. J Clin Microbiol. 2015;53(8):2410-26.

39. Kleinheinz KA, Joensen KG, Larsen MV. Applying the ResFinder and VirulenceFinder. 2014;(December):1-7.

40. Arndt D, Grant JR, Marcu A, Sajed T, Pon A, Liang Y, et al. PHASTER: a better, faster version of the PHAST phage search tool. Nucleic Acids Res. 2016; 44(W1):W16-21.

41. Gray MD, Lampel KA, Strockbine NA, Fernandez RE, Melton-Celsa AR, Maurelli AT. Clinical isolates of shiga toxin 1a-producing Shigella flexneri with an epidemiological link to recent travel to hispañiola. Emerg Infect Dis. 2014;20(10):1669-77.

42. Kozyreva VK, Jospin G, Greninger AL, Watt JP, Eisen JA, Chaturvedi V. Recent Outbreaks of Shigellosis in California Caused by Two Distinct Populations of Shigella sonnei with either Increased Virulence or Fluoroquinolone Resistance. mSphere [Internet]. 2016;1(6):1-18. Available from: http:// msphere.asm.org/lookup/doi/https://doi.org/10.1128/mSphere.00344-16.

43. Croxen MA, Law RJ, Scholz R, Keeney KM, Wlodarska M, Finlay BB. Recent advances in understanding enteric pathogenic Escherichia coli. Clin Microbiol Rev. 2013;26(4):822-80

44. Johnson RP, Holtslander B, Mazzocco A, Roche S, Thomas JL, Pollari F, et al. Detection and prevalence of verotoxin-producing Escherichia coli 0157 and Non-O157 serotypes in a Canadian watershed. Appl Environ Microbiol. 2014; 80(7):2166-75.

45. Ahmed SA, Awosika J, Baldwin C, Bishop-Lilly KA, Biswas B, Broomall S, et al. Genomic Comparison of Escherichia coli 0104:H4 Isolates from 2009 and 2011 Reveals Plasmid, and Prophage Heterogeneity, Including Shiga Toxin Encoding Phage stx2. Ibekwe AM, editor. PLoS One [Internet]. 2012 Nov 1; 7(11):e48228. Available from: https://doi.org/10.1371/journal.pone.0048228.

46. Koutsoumanis K, Allende A, Alvarez-Ordóñez A, Bover-Cid S, Chemaly M, Davies $R$, et al. Pathogenicity assessment of Shiga toxin-producing Escherichia coli (STEC) and the public health risk posed by contamination of food with STEC. EFSA J. 2020;18(1):1-105.
47. Alperi A, Figueras MJ. Human isolates of Aeromonas possess Shiga toxin genes (stx1 and st $\times 2$ ) highly similar to the most virulent gene variants of Escherichia coli. Clin Microbiol Infect [Internet]. 2010;16(10):1563-7. Available from: https://doi.org/10.1111/j.1469-0691.2010.03203.x.

48. Boisen N, Melton-Celsa AR, Hansen A-M, Zangari T, Smith MA, Russo LM, et al. The Role of the AggR Regulon in the Virulence of the Shiga ToxinProducing Enteroaggregative Escherichia coli Epidemic O104:H4 Strain in Mice. Front Microbiol. 2019;10(August):1-11.

49. Beutin L, Hammerl JA, Reetz J, Strauch E. Shiga toxin-producing Escherichia coli strains from cattle as a source of the Stx2a bacteriophages present in enteroaggregative Escherichia coli O104: H4 strains. Int J Med Microbiol [Internet]. 2013;303(8):595-602. Available from: https://doi.org/10.1016/j. ijmm.2013.08.001.

50. Patel PN, Lindsey RL, Garcia-Toledo L, Rowe LA, Batra D, Whitley SW, et al. High-Quality Whole-Genome Sequences for 77 Shiga Toxin-Producing Escherichia coli Strains Generated with PacBio Sequencing. Genome Announc [Internet]. 2018 May 10;6(19):1-4. Available from: http://genomea.a sm.org/lookup/doi/https://doi.org/10.1128/genomeA.00391-18.

51. Feng PCH, Councell T, Keys C, Monday SR. Virulence characterization of Shiga-toxigenic Escherichia coli isolates from wholesale produce. Appl Environ Microbiol. 2011;77(1):343-5.

52. Ha AD, Denver DR. Comparative Genomic Analysis of 130 Bacteriophages Infecting Bacteria in the Genus Pseudomonas. Front Microbiol [Internet]. $2018 \mathrm{Jul}$ 4;9(JUL):1-13. Available from: https://www.frontiersin.org/article/ https://doi.org/10.3389/fmicb.2018.01456/full.

53. Aziz RK, Breitbart M, Edwards RA. Transposases are the most abundant, most ubiquitous genes in nature. Nucleic Acids Res. 2010;38(13):4207-17.

54. Kusumoto M, Nishiya Y, Kawamura Y. Reactivation of Insertionally Inactivated Shiga Toxin 2 Genes of Escherichia coli 0157:H7 Caused by Nonreplicative Transposition of the Insertion Sequence. Appl Environ Microbiol [lnternet]. 2000 Mar 1;66(3):1133-8. Available from: https://aem.a sm.org/content/66/3/1133.

55. Siguier P, Gourbeyre E, Chandler M. Bacterial insertion sequences: Their genomic impact and diversity. FEMS Microbiol Rev. 2014;38(5):865-91.

56. Ooka T, Ogura Y, Asadulghani M, Ohnishi M, Nakayama K, Terajima J, et al. Inference of the impact of insertion sequence (IS) elements on bacterial genome diversification through analysis of small-size structural polymorphisms in Escherichia coli 0157 genomes. Genome Res. 2009; 19(10):1809-16.

57. Mauro SA, Koudelka GB. Shiga toxin: Expression, distribution, and its role in the environment. Toxins (Basel). 2011;3(6):608-25.

58. Evans T, Bowers RG, Mortimer M. Modelling the stability of Stx lysogens. J Theor Biol. 2007;248(2):241-50.

59. Bloch S, Nejman-Faleńczyk B, Dydecka A, Łoś JM, Felczykowska A, Węgrzyn A, et al. Different Expression Patterns of Genes from the Exo-Xis Region of Bacteriophage $\lambda$ and Shiga Toxin-Converting Bacteriophage $\Phi 24 B$ following Infection or Prophage Induction in Escherichia coli. Dąbrowska K, editor. PLoS One [Internet]. 2014 Oct 13;9(10):e108233. Available from: https://doi. org/10.1371/journal.pone.0108233.

60. Bloch S, Nejman-Faleńczyk B, Łoå JM, Barańska S, Łepek K, Felczykowska A, et al. Genes from the exo-xis region of $\lambda$ and Shiga toxin-converting bacteriophages influence lysogenization and prophage induction. Vol. 195, Archives of Microbiology. 2013. p. 693-703.

61. Olavesen KK, Lindstedt BA, Løbersli I, Brandal LT. Expression of Shiga toxin 2 (Stx2) in highly virulent Stx-producing Escherichia coli (STEC) carrying different anti-terminator (q) genes. Microb Pathog. 2016;97:1-8.

62. Fogg PCM, Allison HE, Saunders JR, McCarthy AJ. Bacteriophage Lambda: a Paradigm Revisited. J Virol. 2010;84(13):6876-9.

63. Wang I-N, Smith DL, Young R. Holins: The Protein Clocks of Bacteriophage Infections. Annu Rev Microbiol. 2000;54(1):799-825.

64. Wagner PL, Livny J, Neely MN, Acheson DWK, Friedman DI, Waldor MK. Bacteriophage control of Shiga toxin 1 production and release by Escherichia coli. Mol Microbiol. 2002;44(4):957-70.

65. Unkmeir A, Schmidt H. Structural analysis of phage-borne stx genes and their flanking sequences in Shiga toxin-producing Escherichia coli and Shigella dysenteriae type 1 strains. Infect Immun. 2000;68(9): 4856-64.

66. Teel LD, Melton-Celsa AR, Schmitt CK, O'Brien AD. One of two copies of the gene for the activatable Shiga toxin type $2 \mathrm{~d}$ in Escherichia coli O91:H21 strain B2F1 is associated with an inducible bacteriophage. Infect Immun. 2002;70(8):4282-91. 
67. Mühldorfer I, Hacker J, Keusch GT, Acheson DW, Tschäpe H, Kane AV, et al. Regulation of the Shiga-like toxin II operon in Escherichia coli. Infect Immun. 1996;64(2):495-502.

68. Neely MN, Friedman DI. Functional and genetic analysis of regulatory regions of coliphage $\mathrm{H}$ - 19B: Location of shiga-like toxin and lysis genes suggest a role for phage functions in toxin release. Mol Microbiol. 1998; 28(6):1255-67.

69. Miyamoto H, Nakai W, Yajima N, Fujibayashi A, Higuchi T, Sato K, et al. Sequence analysis of Stx2-converting phage VT2-Sa shows a great divergence in early regulation and replication regions. DNA Res. 1999;6(4): 235-40.

70. Joensen KG, Scheutz F, Lund O, Hasman H, Kaas RS, Nielsen EM, et al. Realtime whole-genome sequencing for routine typing, surveillance, and outbreak detection of verotoxigenic Escherichia coli. J Clin Microbiol. 2014; 52(5):1501-10

71. Gray MD, Lacher DW, Leonard SR, Abbott J, Zhao S, Lampel KA, et al. Prevalence of Shiga toxin-producing Shigella species isolated from French travellers returning from the Caribbean: An emerging pathogen with international implications. Clin Microbiol Infect [Internet]. 2015;21(8):765.e9765.e14. Available from: https://doi.org/10.1016/j.cmi.2015.05.006.

72. Kearse M, Moir R, Wilson A, Stones-Havas S, Cheung M, Sturrock S, et al. Geneious Basic: An integrated and extendable desktop software platform for the organization and analysis of sequence data. Bioinformatics. 2012; 28(12):1647-9.

73. Cresawn SG, Bogel M, Day N, Jacobs-Sera D, Hendrix RW, Hatfull GF. Phamerator: A bioinformatic tool for comparative bacteriophage genomics. BMC Bioinformatics [Internet]. 2011;12(1):395. Available from: http://www. biomedcentral.com/1471-2105/12/395.

74. Huson DH, Bryant D. Application of phylogenetic networks in evolutionary studies. Mol Biol Evol. 2006;23(2):254-67.

75. Johansen BK, Wasteson Y, Granum PE, Brynestad S. Mosaic structure of Shiga-toxin-2-encoding phages isolated from Escherichia coli 0157:H7 indicates frequent gene exchange between lambdoid phage genomes. Microbiology. 2001;147(7):1929-36.

\section{Publisher's Note}

Springer Nature remains neutral with regard to jurisdictional claims in published maps and institutional affiliations.

Ready to submit your research? Choose BMC and benefit from:

- fast, convenient online submission

- thorough peer review by experienced researchers in your field

- rapid publication on acceptance

- support for research data, including large and complex data types

- gold Open Access which fosters wider collaboration and increased citations

- maximum visibility for your research: over $100 \mathrm{M}$ website views per year

At $\mathrm{BMC}$, research is always in progress.

Learn more biomedcentral.com/submissions 